\title{
Smartphone Apps Targeting Alcohol and Illicit Substance Use: Systematic Search in in Commercial App Stores and Critical Content Analysis
}

Babak Tofighi ${ }^{1}$, MD, MSc; Chemi Chemi ${ }^{1}$, MPH; Jose Ruiz-Valcarcel ${ }^{2}$, MPH, MSGIS; Paul Hein ${ }^{1}$, MD; Lu Hu ${ }^{1}$, $\mathrm{PhD}$

${ }^{1}$ Department of Population Health, New York University School of Medicine, New York, NY, United States

${ }^{2}$ University of Puerto Rico School of Medicine, San Juan, Puerto Rico

Corresponding Author:

Babak Tofighi, MD, MSc

Department of Population Health

New York University School of Medicine

227 East 30th Street, Room 713

New York, NY, 10016

United States

Phone: 16468017345

Email: babak.tofighi@nyumc.org

\section{Abstract}

Background: Smartphone apps promise to enhance the reach of evidence-based interventions (cognitive behavior therapy, contingency management and therapeutic education system) for populations with substance use disorders, with minimal disruption to health systems. However, further studies are needed to systematically evaluate smartphone apps targeting alcohol and illicit substances.

Objective: The aim of this study was to evaluate the functionality, aesthetics, and quality of information of free or low-cost apps claiming to target alcohol, benzodiazepine, cocaine, crack/cocaine, crystal methamphetamine, and heroin use using the validated Mobile App Rating Scale (MARS) and critical content analysis.

Methods: A systematic search of iTunes and Google Play app stores for free or low-cost apps facilitating recovery was conducted in March 2018 and yielded 904 apps using the keywords described in previous studies (eg, recovery, sobriety, sober, alcohol, and heroin). An interdisciplinary team of clinicians, behavioral informatics, and public health reviewers trained in substance use disorders conducted a descriptive analysis of 74 apps categorized as reducing use. In addition to the MARS scale, a descriptive analysis of relevant apps was conducted by the study team to assess for quality indicators emphasized by expert guidelines and review articles.

Results: Most apps $(\mathrm{n}=74)$ claimed to reduce use or promote abstinence and yielded an overall low median MARS score of $2.82(0.55)$ and a wide range of scores $(1.64,4.20)$. Ratings were also low for engagement $(2.75(0.72))$, functionality $(3.64$ $(0.78)$ ), aesthetics $(3.03(0.87))$, information (2.82 (0.62)), and satisfaction (1.76 (0.67)) subdomains. Innovative design and content features elicited in the review included initial assessments of substance use following app download, tracking substance use, and related consequences (eg, cost or calorie intake), remote and proximate peer support per geospatial positioning, and allowing users and family members of individuals with substance use disorders to locate 12-step meetings, treatment programs, and mental health services. Few apps integrated evidence-based psychotherapeutic (eg, cognitive behavioral therapy [CBT] or motivational interviewing) and pharmacologic interventions (eg, naloxone or buprenorphine).

Conclusions: Few commercially available apps yielded in our search integrated evidence-based interventions (eg, extended-release naltrexone, buprenorphine, naloxone, Self-Management and Recovery Training recovery, or CBT), and a concerning number of apps promoted harmful drinking and illicit substance use.

(JMIR Mhealth Uhealth 2019;7(4):e11831) doi: $\underline{10.2196 / 11831}$

\section{KEYWORDS}

mHealth; substance use disorder; mobile health; alcohol abuse 


\section{Introduction}

Mobile phone-based health (mHealth) interventions offer a ubiquitous and low-cost approach to improving health outcomes. Smartphone apps, short message service (SMS) text messaging, and interactive voice response are effective approaches to reducing the burden of substance use disorders (SUDs) [1-4]. Most Americans now own smartphones (77\%), and smartphones are especially popular among younger adults aged 18 to 29 years (92\%) [5]. Smartphone apps promise to enhance the reach of evidence-based interventions (cognitive behavior therapy, contingency management, and therapeutic education system) for populations with SUDs with minimal disruption to health systems [2,6-8]. Reports have estimated the availability of over 318,000 mHealth apps in 2017 and the use of health-related apps by approximately half of all smartphone users in 2018 [9]. Considering existing barriers to formal treatment for SUDs, including perceived stigma, cost, and limited treatment slots [10], smartphone apps are increasingly utilized by individuals excluded from care to reduce alcohol and illicit substance use [11-13].

Concerns regarding the quality, efficacy, and privacy of mHealth apps persist. App descriptions routinely include unsubstantiated claims of medical expertise and intervention efficacy, while failing to disclose the sale of health information and personal data gathered from the user to third party vendors for commercial use [14]. Studies assessing mHealth apps targeting SUDs in smartphone app stores (ie, Google Play and/or iTunes) mostly described commercially driven apps that failed to offer evidence-based psychosocial interventions or to link users to addiction treatment providers $[11,12,15]$. In a descriptive analysis of apps addressing alcohol use, Weaver et al also found that apps claiming to inform users of their possible blood alcohol concentration actually promoted risky drinking behavior via games and other entertaining features [12]. Searches also yielded many recreational apps that promoted drug cultivation, trafficking, and simulated use [16].

Despite these findings, further studies are needed to systematically evaluate smartphone apps targeting alcohol and illicit substances. Studies assessing app content are generally descriptive and limited to one-dimensional outcome quality measures [17]. Previous searches of apps targeting SUDs were often limited to a single app provider (iTunes or Google Play), did not use keywords described by users to search for apps targeting SUDs (ie, recovery, sobriety, abstinence, and detox), and did not incorporate validated methods of assessing smartphone apps $[13,14,18]$. In addition, in the last 3 years, as most of these searches were conducted, the number of mHealth apps has doubled from 165,000 apps in 2015 to 325,000 mHealth apps in 2017 [19].

Our study utilized the validated Mobile App Rating Scale (MARS)[18] and critical content analysis [14], which offered a standardized approach to evaluate the functionality, aesthetics, and quality of information of apps claiming to target alcohol, benzodiazepine, cocaine, crack/cocaine, crystal methamphetamine, and heroin use. The MARS is the first mHealth app-quality indicator of engagement, functionality, aesthetics, and informational content delivered by a multidisciplinary team of clinicians and technology experts [18]. Our research also utilized a critical content analysis of apps listed in the smartphone stores claiming to reduce substance use to assess actual clinical impact (ie, review of the literature and app developer website) and linkage to patient-centered care models for addiction treatment (ie, self-efficacy, education, linkage with addiction specialty care, primary care, self-help groups, and/or individual counseling) prioritized by public health experts [20,21].

\section{Methods}

\section{Smartphone App Selection}

In March 2018, a systematic search of smartphone apps facilitating recovery from alcohol and illicit substances was conducted on the iTunes App Store and Google Play (see Figure 1) because of their popularity in the United States for app users. Additional apps targeting alcohol and illicit substance use described in the literature (eg, PubMed, Google Scholar, and PsycInfo) were also included in the search. The keyword search was in English, and apps that met the inclusion criteria were downloaded on an American mobile service network on the iPhone 6, iPhone 7, iPhone 7 plus, Samsung Galaxy J7, and Android LG G6 models.

The search terms were based on relevant terms described in the literature and in previous surveys among participants who utilized smartphone apps to reduce substance use (eg, sober, sobriety, recovery, crystal methamphetamine, opioid, alcohol, cocaine, crack cocaine, and benzodiazepine) [22]. The initial selection of apps excluded apps found to be irrelevant per the app title and online app store description (eg, music/relaxation, games, clocks, and religiosity), apps not in English, apps that lacked accessibility or functionality, apps designed for health care professionals, apps that cost more than \$1 US dollars (on the grounds that they were unlikely to be purchased by a large number of users), and harmful apps promoting substance use. Although 12-step groups are an essential approach in reducing the burden of SUDs across diverse populations with SUDs [23], apps based solely on the 12-step model were not reviewed because of the large quantity of apps and limited study resources and their exclusion of content pertaining to medication-assisted treatments and effective behavior change models outlined by federal and expert guidelines [24]. The total number of apps yielded from our search was similar to previous studies on recovery apps $[11,12,15]$. Owing to the large number of apps retrieved in our initial search, apps without any user star ratings or reviews were also excluded in the preliminary screening. Apps that met preliminary inclusion criteria were then downloaded to the coauthors' smartphones and assessed for accessibility, functionality, and relevancy of app content to reducing substance use (see Figure 1). 
Figure 1. Flow diagram for smartphone app selection.

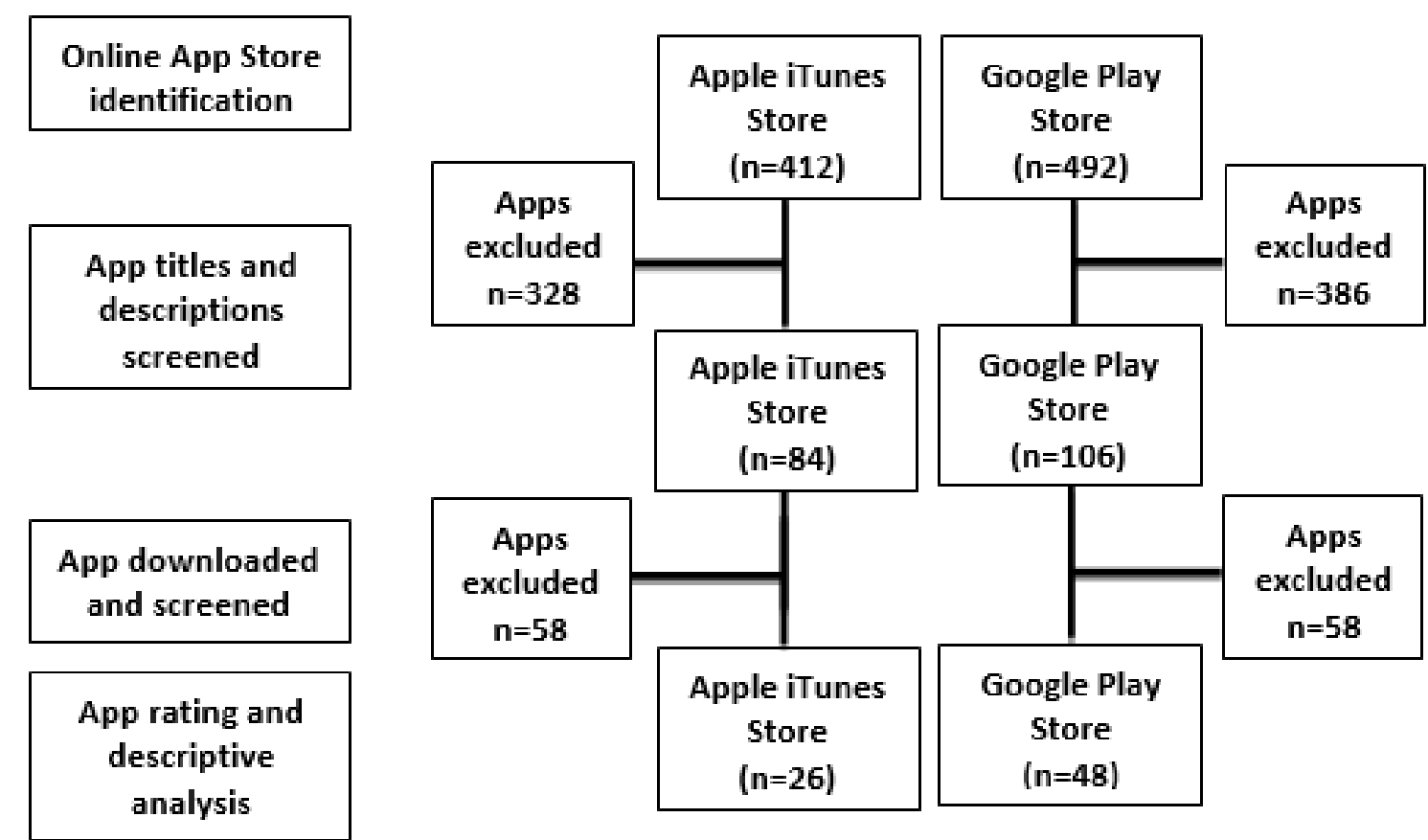

\section{App Assessment}

App quality was assessed using the MARS [18]. The MARS domains (ie, engagement, functionality, aesthetics, information quality, and subjective quality) are constituted by 23 items based on a 5-point scale (1-Inadequate, 2-Poor, 3-Acceptable, 4-Good, and 5-Excellent). The classification domain is a descriptive survey of app price, platform, rating, and technical features (eg, password protection and log-in protocols). The excellent internal consistency (alpha=.92) and interrater reliability (intraclass correlation, ICC $=.85$ ) of the MARS scale make it ideal for conducting initial assessments of emerging apps [18]. Apps are then scored by calculating the mean scores of each respective subscale and the total mean score. The MARS scale has been successfully utilized to assess apps targeting a range of health conditions, including asthma, heart failure, and cancer [25-28]. However, the MARS scale has not been used to assess the quality of smartphone apps targeting alcohol and illicit substance use.

To ensure shared understanding of review criteria and MARS subscales, the study team reviewed the MARS literature and discussed each domain and subscale. The interdisciplinary study team included clinicians with experience in SUDs (BT and $\mathrm{PH}$ ) and mHealth design (BT, PH, JRV, and LH). The reviewers convened and used 3 separate meetings to pilot apps and compare ratings per the MARS scale, assess the quality of their ratings, and resolve any discrepancies and ambiguities in the scale items. Each app was tested for at least $15 \mathrm{~min}$. The raters then independently assessed 5 apps, and these apps were used to test the MARS tool, compare our judgments, and resolve any discrepancies. Interrater reliability among iTunes app raters was high $(\mathrm{ICC}=.80)$, but the overall app MARS score had fair interrater reliability ( $\mathrm{ICC}=.58$ ) requiring additional individual and group meetings to address discrepancies that emerged during the initial review. The study team compared ratings using iTunes for pragmatic reasons as most of the raters were Apple smartphone owners.

In the final round, we reviewed a total of 74 apps (26 Apple and 48 Google Play apps). Apps that met the inclusion criteria were assessed individually by the study authors on their smartphones in April 2018. In addition to the MARS scale, a descriptive analysis of relevant apps was conducted by the study team to assess for quality indicators emphasized by expert guidelines [8,29], as well as review articles [4,17,30], as evidence-based design practices in mHealth interventions targeting SUDs. This descriptive analysis elicited critical findings in app design and delivery features including (1) information or access to medication-assisted treatments via primary care and specialty addiction treatment programs (ie, residential treatment and intensive outpatient programs); (2) risk-reduction content (eg, safe sex practices, syringe exchange programs, naloxone, $\mathrm{HIV}$, and hepatitis $\mathrm{C}$ virus [HCV] prevention education); (3) integration of behavior-change content in the intervention design or content (eg, cognitive behavioral therapy [CBT], motivational enhancement therapy, and contingency management); (4) empirical evidence demonstrating smartphone app efficacy by conducting searches in online databases (eg, PubMed and Google Scholar); and (5) privacy measures (eg, password protection, email or text verification, and user de-identification). Negative features of the reviewed apps assessed for the use of disruptive or distractive ads, religious texts, content exacerbating substance use (ie, access to discount liquor and bars with drink specials), and unverified claims of professional and clinical legitimacy. 
Finally, we identified apps that were aligned with the National Institute on Drug Abuse's strategic objective of enhancing chronic disease management and personalized treatment that matches an addicted person's changing needs over time based on the Medical Management outline (ie, patient-provider communication, medication adherence, self-management, goal of opioid abstinence, and counseling participation) to improve linkage of office-based opioid treatment with effective pharmacotherapies (ie, buprenorphine-naloxone and extended-release naltrexone) [31-34]. During the final round of reviews of apps that met the eligibility criteria, the primary author reviewed $30 \%$ of the apps individually assessed by the secondary authors to ensure consistency with the MARS subscales and descriptive analysis guidelines.

\section{Results}

\section{Summary of Findings}

The Google and iTunes searches identified a total of 904 apps of which 412 were on iTunes and 492 from Google Play (see Figure 1). The initial review excluded apps that were irrelevant $(n=322)$, required payment for use $(n=184)$, were linked with harmful use (eg, gamification of binge drinking or simulating illicit substance use; $n=118)$, were duplicates $(n=70)$, and were not in English $(\mathrm{n}=20)$.

Our secondary review then assessed a total 84 Apple apps and 106 Google apps that met the inclusion criteria. Apps were assessed individually by the study team, and 116 apps were excluded owing to the following factors: (1) unavailability in the app stores 3 weeks between the initial and secondary reviews $(\mathrm{n}=32)$; (2) duplicates $(\mathrm{n}=18)$; (3) educational content for health care providers $(n=16) ;(4)$ required payments for full use of the app $(n=9) ;(5)$ focused exclusively on the 12-step approach $(n=9) ;(6)$ irrelevant to recovery $(n=8) ;(7)$ not functioning $(n=5)$; (8) required invitation from a treatment program $(n=4)$; (9) required a device purchase (eg, breathalyzer; $n=4) ;(10)$ only displayed a clock without any recovery content $(n=4) ;(11)$ were not functional $(n=3)$; (12) only offered religious content $(n=2)$; (13) not in English $(n=1)$; and (14) exacerbated harmful use $(\mathrm{n}=1)$.

Of the remaining apps, 74 met the inclusion criteria and underwent further analysis.

Most apps targeted alcohol use $(n=40)$. Fewer apps emerged from this review exclusively addressing opioid use $(n=6)$ and none focused on cocaine, crack/cocaine, or methamphetamine use. The overall median score of apps included in this study was poor $(2.82(0.55))$ based on the 5-point MARS scale and demonstrated a wide range of ratings $(1.64,4.20$; see Table 1$)$. Scores for engagement (2.75 (0.72)), functionality (3.64 (0.78)), aesthetics (3.03 (0.87)), information (2.82 (0.62)), and satisfaction $(1.76(0.67))$. Apps that had the highest average MARS score included SoberWorx (4.20), Recovery Today Magazine (3.77), Sober Grid (3.75), and Addicaid: Addiction Recovery and Support (3.64). The lowest-ranking apps included Sick Not Stupid (1.64), Sober Day Recovery App (1.71), and Stop Drinking Alcohol Now (1.75).

\section{Innovative Features of High-Quality Apps Targeting Substance Use Disorders}

Use of the MARS scale and descriptive analysis allowed the study team to evaluate higher-rated apps (see Table 1) for design features that may be attributed to increased user engagement and potential clinical impact. Innovative design and content features elicited in the review included initial assessments, tracking substance use and related consequences (eg, cost and calorie intake), remote and proximate peer support per geospatial positioning, and allowing users and family members of individuals with SUDs to locate 12-step group meetings, treatment programs, and mental health services. Apps commonly elicited initial assessments of substance use patterns among users following app download. However, the only 2 apps that utilized an evidence-based approach to initial assessment were The Saying When and Alcohol Tracker apps.

The Saying When app (3.33) was developed by The Canadian Centre for Addiction and Mental Health to facilitate abstinence or reductions in the drinking quantity. The app features include an initial baseline assessment of drinking patterns, setting personalized goals, tracking drinks and urges, offering tips for success, and linkage to community treatment services. The self-help approach of the Saying When app was adapted from a manualized version based on cognitive and behavioral strategies that were not elaborated in the app store description and accompanying Web page. No scientific publications related to the app were available. However, app components appeared to be a cross-over of interventions described by the authors in earlier studies as having clinical impact in reducing drinking, including (1) exposure to initial assessments on drinking patterns [35] and (2) receipt of self-help books outlining initial assessments of drinking combined with the manualized version [36].

Alcohol Tracker (2.97) utilized the Alcohol Use Disorders Identification Test and Functional Analysis of Addictive Behaviors to notify users if they have surpassed alcohol consumption based on the National Institute for Health and Care Excellence UK Guidelines and link users to treatment resources. However, only users based in Singapore can access hotlines. In addition, reliance on self-reported drinking by apps tracking use is at risk of recall bias, particularly during binge-drinking episodes. 
Table 1. Mobile app rating scale results. App availability is subject to removal per app developers, Google Play, and the Apple iTunes Store. Apps included in this table were rated as $>3$ or higher.

\begin{tabular}{|c|c|c|c|c|c|c|}
\hline Smartphone app name & Engagement & Functionality & Aesthetics & Information & Satisfaction & Overall Score \\
\hline SoberWorx & 4.4 & 4.5 & 5 & 3.83 & 3.25 & 4.2 \\
\hline Recovery Today Magazine & 3.8 & 4.5 & 4.3 & 3.5 & 2.75 & 3.77 \\
\hline Sober Grid & 3.8 & 3.25 & 4.3 & 3.67 & 3.75 & 3.75 \\
\hline Addicaid: Addiction Recovery Support & 3.8 & 4 & 4.67 & 3.5 & 2.25 & 3.64 \\
\hline BoozeFit & 3.2 & 4.5 & 4 & 3.5 & 2 & 3.44 \\
\hline CleanTime Counter & 2.2 & 4 & 4.25 & 3.5 & 3 & 3.39 \\
\hline Wise Drinking & 3.2 & 3.5 & 4.667 & 2.83 & 2.75 & 3.39 \\
\hline SoberApp-Alcohol Calculator & 3.4 & 4 & 3.33 & 3.67 & 2.5 & 3.38 \\
\hline Alcohol Check - BAC Calculator & 3.4 & 3.5 & 4.3 & 3.4 & 2.25 & 3.37 \\
\hline FlexDek: Anglestrong Edition & 3.8 & 4 & 3.3 & 3.16 & 2.5 & 3.35 \\
\hline Saying When & 3.4 & 3.5 & 4 & 3.5 & 2.25 & 3.33 \\
\hline OARS Experience & 2.8 & 3.75 & 2.5 & 4 & 3 & 3.21 \\
\hline Drinks Meter & 2.75 & 4.25 & 2.67 & 3.5 & 2.75 & 3.18 \\
\hline Drive Sober & 3.8 & 4.75 & 2.3 & 3.3 & 1.75 & 3.18 \\
\hline Best Alcohol Test & 3.6 & 3.75 & 3.5 & 3 & 2 & 3.17 \\
\hline Stop OD NYC & 2 & 4.25 & 3.3 & 3.28 & 3 & 3.17 \\
\hline Drug Addiction Recovery & 2.6 & 3.75 & 4.3 & 3.6 & 1.5 & 3.15 \\
\hline Blood Alcohol Content Calculator+Timer & 2.6 & 4.75 & 3.3 & 3 & 2 & 3.13 \\
\hline Alcohol Tracker & 2.6 & 4.25 & 3.6 & 3.4 & 1.75 & 3.12 \\
\hline My Drink Control & 3 & 4.25 & 3 & 3.6 & 1.75 & 3.12 \\
\hline AlcDroid Alcohol Tester & 3 & 4.25 & 2.67 & 3.16 & 2.5 & 3.12 \\
\hline BACTrack & 3.4 & 4.5 & 3.66 & 2.5 & 1.5 & 3.11 \\
\hline Sober Grid & 3.75 & 3.3 & 3 & 3 & 2.5 & 3.11 \\
\hline Clean \& Sober Time & 3 & 3.5 & 4 & 3 & 2 & 3.1 \\
\hline Alcohol Calorie Counter & 2.6 & 3.75 & 4.67 & 2.75 & 1.5 & 3.05 \\
\hline Intoxication Calculator & 3.4 & 3.4 & 3.3 & 3.4 & 1.75 & 3.05 \\
\hline Addiction Quotes & 3.2 & 4 & 3.3 & 3 & 1.75 & 3.05 \\
\hline Habit Tracker & 2.6 & 4 & 3.3 & 3 & 2.25 & 3.03 \\
\hline Wbi.today & 3.6 & 3.25 & 3.3 & 3.16 & 1.75 & 3.01 \\
\hline
\end{tabular}

Several apps claimed to provide users access to sober peers via intra-app messaging, help icons, or forums. The most intriguing app (MARS score 3.75) facilitating peer support was Sober Grid and was developed by a team of academics and developers to offer a global newsfeed of shared posts on experiences in and insights into recovery and an instant help feature to link users to available peers online and in-person. The app then encourages patients to refer actively using peers to treatment. The app also encourages adoption among clinicians and health systems by offering an administrator dashboard, the option to launch mass notifications and messages to patients, and onboarding support. In addition, health systems may use the app to track substance use among patients during and post treatment and allow patients to meet and provide online support.
Addicaid Recovery Support (3.64) also offers linkage to peers in recovery and self-help group meetings. Some of the topics include mothers in recovery, adult children of alcoholics, narcotics, methamphetamine, and heroin. However, once a user joins the group, the posts are all from over a year ago. There are also a series of sessions such as starting recovering, commitment and community, introducing a new routine, and recovery maintenance and under each of these goals are comments including positive words of encouragement from peers. Once enabled, the app also provides users with a list of groups and meetings nearby.

Pocket Rehab facilitates text, telephone call, and video conferencing calls with other peers in recovery. Users are able to enter their location to search for nearby 12-step group meetings, a photo motivating sobriety, and an anticipated quit 
date. However, more interestingly, the app offers a Community chat forum with features similar to a Facebook wall and an immediate helpline linking individuals to other sober peers who are using the app and willing to communicate with the user to provide support. Users may select individual or community support and communicate via SMS text messaging, voice, or video. If this contact was not helpful, the app then links users to chat with an experienced peer or write a journal entry. Although the app promises to link individuals to other users within 1 to 2 min, our study team was unable to establish contact with any app user. The app requested access to users' locations and assured that their information would not be available to third-party vendors. The study team gave a lower rating to the app (MARS score 2.66) owing to the lack of peer contact, linkage to behavioral health specialists in addition to peers in recovery, and integration of any evidence based-content.

Additional apps utilized fellow users to provide support. SoberWorx received a rating of 4.20 and was established by individuals in recovery to provide peer support, link to treatment resources, and also allow family members to locate treatment resources for loved ones with SUDs. Although the app claims to offer SMS text messaging-based contact with treatment programs via the app platform, we were unable to communicate with any providers or program staff. In addition to linking users to treatment centers, addiction counseling, and sober living homes, the app also allows for peer support, posting testimonials, and access to educational recovery content via YouTube videos. However, the peer support feature was also not interactive and was also difficult to identify online users and initiate contact.

The only app that clearly demonstrated application of an evidence-based psychotherapeutic approach was the Self-Management and Recovery Training (SMART )Recovery Cost Benefit Analysis (MARS rating 2.85). This app is based on the SMART recovery model, which integrates CBT to offer coping strategies for individuals in recovery to reduce the risk of relapse. The app allows users to enter the costs of ongoing use and the financial benefits of abstinence. However, the poor design (2) and lack of satisfaction (2) by the study team following app use resulted in a lower overall score. Despite the increasing popularity and clinical benefit of engaging with SMART recovery groups, counselors, and educational content based on the SMART platform [37], the app fails to effectively translate these resources into accessible and user-tailored features.

\section{Government-Sponsored Smartphone Apps}

Our search yielded few government-initiated apps and apps that offered risk reduction measures for individuals with SUDs. The most intriguing app was STOP OD NYC (3.17), developed by the New York City Department of Health and Mental Hygiene, which provides detailed information on opioids (eg, heroin and fentanyl) and instructions on naloxone administration in the event of an overdose. The app provides several risk-reduction content, including (1) a find naloxone option that links users to mapped pharmacies, harm reduction programs, and health care centers providing free naloxone; (2) naloxone administration instructions for intramuscular, intranasal, and auto-injector formulations of naloxone; (3) recognizing individuals suspected of an overdose; and (4) information on legal protection for individuals administering naloxone. The app utilizes SMS text messaging, cartoon, and YouTube-based videos to offer users multimedia educational instructions. Finally, users can click on the NYC Health icon to access other health resources within the Department of Health and Mental Hygiene platform (eg, cardiovascular health, reducing glucose intake, and smoking cessation).

My Drink Control (3.12) is an app and Web-based tool developed by the Public Health Department in Zurich, Switzerland, to facilitate tracking of alcohol use, financial costs of use, and calories gained with each drink. Although the app's psychoeducational content was based on CBT, our review of the app failed to elicit any theory-based content or design features. The app's overall functionality was limited to tracking drinks, reminders, and linkage to treatment and counseling services for alcohol use. However, compared with other government-sponsored apps, My Drink Control had a more appealing and user-friendly design.

Furthermore, 1 app designed for the Substance Abuse and Mental Health Services Administration (SAMHSA) app challenge, FlexDek MAT (2.90), described itself as linking participants to the methadone maintenance program but again failed to offer accurate and updated contact information regarding the Office based opioid treatment (OBOT) programs after this feature was utilized. Information pertaining to MAT, including buprenorphine, methadone, MAT, naltrexone, and after naltrexone, was limited to PDF files, links to SAMHSA's website, and an external website [38]. Other links to the 12-step and SMART recovery groups were not functioning after linking to an external website. The forum icon opened an error page and the Coaches option linked users to only 5 recovery coaches across the nation with a nonfunctioning link icon. The rewards option for using the app claimed to offer free hours of recovery coaching, but this was not evident in our review. In addition to linking to nonfunctioning pages, the app also exposed users to irrelevant ads in the lower segment of the screen.

\section{Features of Low-Quality Apps Targeting Substance Use Disorders}

Lower quality apps often claimed to support recovery through complex design features but were limited to 1 to 2 basic functions such as supportive quotes, timers, blood alcohol calculators, or logging daily substance use without providing tailored feedback and evidence-based interventions. Some apps only copied quotes from religious texts without specifying content in the title or description (eg, OARS experience). Furthermore, 1 app entitled Alcoholism Treatment would only play ambient electronic music that app developers claimed would stimulate desires to quit.

Many of these apps used deceptive descriptions of complex recovery resources but would inundate users with pop-up advertisements (eg, Stop Drinking Alcohol Now and Sobriety Clock), require users to log-in via Facebook or Google with access to their social networks, and request access to user location. Among blood alcohol calculators, most lacked useful information on evidence-based treatment approaches and were often rated poorly by our study team and app store users. In 
addition, 1 app, BACtrack, developed by the San Francisco-based BACtrack, enables users to measure blood alcohol levels. However, to utilize the app, users were required to purchase a breathalyzer ( $\$ 99.00$ US dollars) to fully utilize the platform. Users were instructed to breathe into the breathalyzer and promised to log results wirelessly to their mobile device. The only free and functioning feature on the app was a link to Uber to access a ride. Additional apps also claimed to have initiated supportive peer networks, but forums and peer-messaging functions would typically be inactive. Others would link users to 12-step group meeting schedules or 12-step-based online forums rather than the app's own support networks. Motivational content would typically appear in the form of quotes without any integration of behavior-change principles (eg, Recovery Quotes, Clean \& Sober Recovery, and Addiction Quotes)

Other features of low-quality apps included the use of basic functions such as motivational quotes, timers, or informational content to ultimately expose users to pop-up advertisements for a single private residential treatment program or clinician (eg, SoberBud, Sobriety Clock, and Stop Drinking Now). Other apps would solicit users to pay for the full-version app to receive more comprehensive recovery content (eg, Hypnosis for Alcoholism, Addiction and Recovery, and Sober Tree).

Some apps were concerning for the possibility of exacerbating substance use. The Drugs app offered informational content that was not easily accessible, and the forum included threads with individuals offering to sell drugs. The Drive After: Alcohol calculator app offered users information on strategies to feel more sober or conceal the odor of alcohol. Best Alcohol Test is intended to offer a blood alcohol level calculator but also offers users games to test their reflexes following binge-drinking episodes.

Another app described as Best Home Cure for Alcoholism (2.38) claimed to offer alternative remedies for alcohol use and withdrawal symptoms with topics such as how to make someone stop drinking alcohol forever; how to quit alcohol ayurvedic; and how to stop alcohol drinking of my husband. However, content was limited to increasing intake of fruits, vegetables, juices, water, and coffee without offering any empirical evidence on these approaches or elaborating on the credibility of the article authors and app designers.

\section{Discussion}

\section{Summary}

The initial search for apps targeting substance use yielded 74 apps; however, only 7 apps offered any evidence-based content, such as information on effective pharmacotherapies for SUDs $(n=3)$, harm-reduction content $(n=1)$, or behavior-change principles within app content or design features $(n=3)$. None of the apps facilitated linkage to primary care-based treatment for SUDs, methadone maintenance treatment programs for Opioid use disorder (OUD), or clarified insurance requirements or costs related to available primary care or specialty addiction treatment programs.
Although none of the apps cited any empirical evidence suggesting potential clinical benefit or sustained engagement among users, app quality assessment via the MARS score offers a useful approach before evaluating for efficacy. The apps in this study had a low overall median quality MARS score of 2.81. However, the overall low information (2.81), engagement (2.75), and satisfaction (1.75) subscale scores highlight the lack of evidence-based content and gap in intervention design.

Although numerous apps emerged from the review targeting alcohol use, only STOP OD NYC and FlexDek MAT specifically targeted opioid use with evidence-based content (eg, effective pharmacotherapies for OUD). However, STOP OD NYC was limited to naloxone and overdose prevention and FlexDek MAT lacked basic functionality, was not aesthetically engaging, and the informational content was limited to PDF files and external links to the SAMHSA Web page. None of the commercially developed apps offered any informational content or access to online or clinical resources addressing harm-reduction practices and HIV and $\mathrm{HCV}$ prevention or screening content.

Few apps integrated evidence-based behavior change content (eg, SMART Recovery Cost Benefit) and mostly centered on basic informational content summarizing how many calories or money would be saved with alcohol abstinence, tracking time of abstinence with timers, using graphs to chart quantities of consumed alcoholic beverages, and basic information about addiction (eg, as a chronic disease), improvised tips on recovery, or quotes from the Bible that were not aligned with evidence-based psychotherapeutic approaches.

\section{Empirical Evidence Demonstrating Smartphone App Efficacy}

Although smartphone software apps are technologically capable to enhance care for SUDs with complex and multifaceted interventions, our review found that even apps with higher MARS ratings were typically limited to singular functions (eg, validated assessments such as Alcohol Use Disorders Identification Test (AUDIT) or Alcohol, Smoking and Substance Involvement Screening Test (ASSIST), active peer support systems, access to 12-step or SMART recovery group meetings, and/or linkage to specialty addiction treatment providers).

In a similar review of alcohol-related smartphone apps in 2012 by Weaver et al, only 44 of the 500 apps that met the initial inclusion criteria actually targeted reductions in alcohol use, and none presented evidence of efficacy [12]. Despite a doubling in apps targeting alcohol use in a subsequent review in 2014, there was no evidence of improved integration of evidence-based approaches (eg, effective psychotherapeutic interventions) and most promoted alcohol use [39]. Our review of commercially available alcohol-reduction apps is aligned with previous findings limiting their use in real-world clinical settings and is concerning for exacerbating relapse or worsening alcohol use [12,32].

MARS ratings of commercially available apps in this study also parallel the critical analysis of apps targeting heart failure and mindfulness and their lack of evidence-guided content $[28,40]$. These findings contrast with randomized controlled trials 
demonstrating effectiveness for university-developed smartphone apps targeting alcohol use [2,41] and cravings $[7,42]$. However, our review found that these apps remain unavailable for individual use via existing app stores and without invitation by a licensed addiction treatment provider.

Although the apps lacked any clinical evidence of efficacy, there was also no evidence of sustained use. For instance, apps claiming to offer peer support via forums or SMS text messaging contact with other users were not active or unresponsive. In addition, forums lacked moderators or clinicians who could offer evidence-based responses to forum threads. Long-term engagement with technology-based interventions is critical to ensure behavior change and meaningful clinical outcomes. Challenges to larger-scale adoption of mHealth interventions include the lack of open mHealth frameworks that clarify underlying mechanisms linking intervention design features, effective psychotherapeutic approaches, and clinical outcomes. For instance, even basic process measures, such as the duration or intensity of app utilization, are not assessed or disclosed. Instead, users and researchers alike must rely on other user ratings and comments to gauge an app's potential benefit. Thus, preliminary studies are needed among participants in the community or addiction treatment settings assessing the impact of self-reported app usage targeting substance use and treatment utilization. Future smartphone app research requires elucidation of how app design features, content, and behavior-change principles impact targeted clinical outcomes. In July 2017, the Food and Drug Administration (FDA) launched the Digital Health Innovation Action Plan to offer additional oversight over clinical and patient decision support software. Further oversight by the FDA may facilitate transparency in app design, promotion of clinical studies assessing app safety and effectiveness, and increase the confidence of users and health systems for broader adoption [43].

\section{Harm Reduction or Exacerbating Harmful Use?}

Apps addressing alcohol use linked participants to app-based transportation companies or sober peers to facilitate rides if they were intoxicated. Some of the apps offering blood alcohol calculators for alcohol encouraged users to avoid reaching intoxication or hazardous drinking levels. However, other blood alcohol calculator apps would use cartoon imagery to gamify drinking and even allow users to compare their blood alcohol levels with other drinking peers. Another blood alcohol calculator app would offer users information about how to hide odors of alcohol in their breath or sober up if they exceeded certain levels of drinking. Our findings are aligned with reviews of smartphone apps in the last decade, emphasizing the availability of commercially developed apps that mostly exacerbate rather than mitigate harmful substance use $[12,16,39]$. Studies have reported the dramatic rise of apps promoting cigarette smoking [44], cannabis [15], and alcohol use [12,39]. In 2012, Bindhim et al searched for cannabis, weed, marijuana, cocaine, heroin, and ecstasy and reported an increase in harmful apps from 238 apps in February 2012 to 410 apps in May 2012 that encouraged contact with actively using peers, role-playing as cartel bosses or cannabis farmers, or simulating substance use [16]. Not surprisingly, in 2017, Google blocked approximately 700,000 of the nearly 3.5 million Android apps purged for promoting violence, hate, adult material, illicit activities, and substance use [45]. However, with 200 new apps entering the marketplace daily [19], app stores must develop more stringent restrictions considering the consistent growth in apps encouraging harmful substance use.

\section{Linkage to Treatment}

Although numerous descriptions attracted potential users to the app's capacity to link them to nearby treatment programs, nearly all lacked updated information about available programs, were not tailored to uninsured or Medicaid-insured patients, or would direct users to a single private practice therapist or residential treatment program even if the investigators were attempting to request an office-based opioid treatment program. The deceptive referral of all requests for treatment to a commercial advertisement or to a single private practice was common. Among apps developed by government agencies, users were able to eventually locate treatment programs after being redirected to government Web pages that listed available clinics but were not adapted for mobile phone-based internet browsers (eg, STOP OD NYC and Drive Sober Alabama). However, the STOP OD NYC app's overall design is the most ideally suited platform to integrate harm-reduction resources (ie, syringe exchange programs and naloxone) as well as linking users to low-cost office-based opioid treatment programs for buprenorphine and extended-release naltrexone treatment and the high density of 12-step and SMART recovery groups within New York City.

\section{Integration of Behavior-Change Content}

CBT and relapse prevention strategies offer a collaborative, individualized, psychological treatment recognized as effective approaches to generating behavioral, cognitive, and emotional adaption to a wide range of common psychological problems [46]. The efficacy of CBT and relapse prevention strategies has been supported by a comprehensive review of 106 meta-analyses across different clinical groups that also extends to SUDs. Despite their widespread adoption in academically developed smartphone apps (eg, A-CHESS), only the SMART cost-benefit app utilized behavior change models in this review.

SMART Recovery is based on both the Rational-Emotive-Behavior therapy and CBT approaches and reinforces learning skills to cope with (rather than avoid) emotional disturbances that exacerbate substance use $[37,46]$. The SMART Recovery website offers extensive resources, including articles, podcasts, videos, and self-help assignments that deepen user engagement with this approach. In addition, its online forum is active and offers unique discussion threads, including Building and Maintaining Motivation, Coping with Urges, Managing Thoughts, Feelings, and Behaviors, Living a Balanced Life, and specialized peer support group forums based on specific substances and a family and friends forum. The Smart Cost Benefit app has tremendous potential to integrate an already vibrant online forum and evidence-based psychotherapeutic approach.

Participation in self-help support groups, online forums, and even smartphone app communities can help motivate users to engage in healthy activities. A supportive app community can 
help users share and discuss their recovery experiences and the challenges of regular practice. This could potentially complement or substitute for the support provided in face-to-face recovery treatment modalities. Although nearly many of the reviewed apps provided social network-sharing, few offered moderators or clinicians to guide discussions. Further research is needed to assess the impact of app-based forums and peer SMS text messaging to enhance engagement with the app and clinical outcomes.

\section{Privacy Measures}

Apps generally lacked the use of privacy measures to protect health information: few required password protection, elucidated the use of security certifications from cellphone providers, utilized encryption technologies for transmitted content, or 2-step verification during registration. Numerous apps asked participants for access to their Facebook profile and/or Google profile during registration and reassured users in the Terms and Conditions that their personal information would be safeguarded. Several apps were also used for research purposes but did not specify details of the research study, rights as a study subject, and how to terminate one's participation in the study and remove their data usage information. The Health Insurance Portability and Accountability Act and expert guidelines have outlined several measures to ensure the privacy of patient-physician communication in emerging health information technologies, including (1) the use of simple message content that refrains from disclosing patient name, diagnosis, or enrollment in treatment; (2) encouraging 2-step verification, password protection, and finger Touch identification; (3) obtaining security certifications from cellphone providers; (4) using encryption technologies for user responses; and (5) regularly deleting or setting expiration periods for communication content [47].

\section{Limitations}

This is one of the first comprehensive studies to review apps targeting illicit substances using the MARS scoring criteria and provide a reliable measure of engagement, functionality, visual appeal, and informational quality. Furthermore, this is the only review to assess for the integration of evidence-based psychotherapeutic or pharmacological approaches to SUDs and their applicability to office-based management of SUDs based on the medical management model. However, findings emerging from our descriptive analysis are not based on validated usability and/or efficacy study methods and require more rigorous study methods to assess the clinical impact. The review was limited to Google Play and Apple iOS and did not include apps available in F-Droid, Amazon Appstore, and GetJar, among other smaller scale app platforms. Our assessment of user privacy did not incorporate open-source developer codes for malicious purposes.

\section{Conclusions}

Online app stores offer unprecedented opportunities to expand access to effective harm reduction and treatment approaches for individuals with SUDs. However, the overall low MARS scale ratings and findings emerging from our descriptive analysis highlight the lack of evidence-based apps for individuals seeking additional support. Further studies are needed to assess the impact of existing evidence-based apps described in this review (eg, STOP OD NYC and FlexDek MAT). Investigators should leverage online app stores to assess the acceptability and clinical impact of effective apps targeting SUDs that are not yet available within online app stores for individual use (eg, A-CHESS). Finally, public health experts should utilize the popularity of online app stores to offer user-friendly and evidence-based apps that facilitate access to effective pharmacotherapies for SUDs, harm-reduction resources (eg, naloxone and syringe exchange programs), specialty addiction treatment programs (eg, intensive outpatient programs and methadone maintenance programs), and linkage to primary care-based treatment for SUDs.

\section{Acknowledgments}

BT is supported by an NIH Mentored Patient-Oriented Research Career Development Award (NIDA K23DA042140-01A1) and Clinical Translational Science Award (UL1 TR001445).

\section{Authors' Contributions}

BT, CC, LH, JRV, and PH made substantial contributions to conception, design, and writing of the manuscript.

\section{Conflicts of Interest}

None declared.

\section{References}

1. Whittaker R, McRobbie H, Bullen C, Rodgers A, Gu Y. Mobile phone-based interventions for smoking cessation. Cochrane Database Syst Rev 2016 Apr 10;4:CD006611. [doi: 10.1002/14651858.CD006611.pub4] [Medline: 27060875]

2. Gustafson DH, McTavish FM, Chih M, Atwood AK, Johnson RA, Boyle MG, et al. A smartphone application to support recovery from alcoholism: a randomized clinical trial. JAMA Psychiatry 2014 May;71(5):566-572 [FREE Full text] [doi: 10.1001/jamapsychiatry.2013.4642] [Medline: 24671165]

3. Brendryen H, Kraft P. Happy ending: a randomized controlled trial of a digital multi-media smoking cessation intervention. Addiction 2008 Mar;103(3):478-84; discussion 485. [doi: 10.1111/j.1360-0443.2007.02119.x] [Medline: 18269367] 
4. Tofighi B, Nicholson JM, McNeely J, Muench F, Lee JD. Mobile phone messaging for illicit drug and alcohol dependence: a systematic review of the literature. Drug Alcohol Rev 2017 Dec;36(4):477-491 [FREE Full text] [doi: 10.1111/dar.12535] [Medline: 28474374]

5. Smith A. Pew Research Center. 2017. Record Shares of Americans Now Own Smartphones, Have Home Broadband URL: http://www.pewresearch.org/fact-tank/2017/01/12/evolution-of-technology/ [accessed 2019-03-15] [WebCite Cache ID

76suJpXmK]

6. Quanbeck AR, Gustafson DH, Marsch LA, McTavish F, Brown RT, Mares M, et al. Integrating addiction treatment into primary care using mobile health technology: protocol for an implementation research study. Implement Sci 2014 May 29;9:65 [FREE Full text] [doi: 10.1186/1748-5908-9-65] [Medline: 24884976]

7. Fowler LA, Holt SL, Joshi D. Mobile technology-based interventions for adult users of alcohol: a systematic review of the literature. Addict Behav 2016 Nov;62:25-34. [doi: 10.1016/j.addbeh.2016.06.008] [Medline: 27310031]

8. Marsch LA. Leveraging technology to enhance addiction treatment and recovery. J Addict Dis 2012;31(3):313-318 [FREE Full text] [doi: 10.1080/10550887.2012.694606] [Medline: 22873192]

9. IQVIA. 2017 Nov 7. The Growing Value of Digital Health URL: https://www.iqvia.com/institute/reports/ the-growing-value-of-digital-health [accessed 2019-03-13] [WebCite Cache ID 76qc4ZE52]

10. Duncan LG, Mendoza S, Hansen H. Buprenorphine maintenance for opioid dependence in public sector healthcare: benefits and barriers. J Addict Med Ther Sci 2015;1(2):31-36 [FREE Full text] [doi: 10.17352/2455-3484.000008] [Medline: 27088135]

11. Penzenstadler L, Chatton A, Van Singer M, Khazaal Y. Quality of smartphone apps related to alcohol use disorder. Eur Addict Res 2016;22(6):329-338. [doi: 10.1159/000449097] [Medline: 27598779]

12. Weaver ER, Horyniak DR, Jenkinson R, Dietze P, Lim MS. “Let's get wasted!” and other apps: characteristics, acceptability, and use of alcohol-related smartphone applications. JMIR Mhealth Uhealth 2013;1(1):e9 [FREE Full text] [doi: 10.2196/mhealth.2709] [Medline: 25100681]

13. Tofighi B, Leonard N, Greco P, Hadavand A, Acosta MC, Lee JD. Technology use patterns among patients enrolled in inpatient detoxification treatment. J Addict Med 2018 Dec 20 [FREE Full text] [doi: 10.1097/ADM.0000000000000494] [Medline: 30589653 ]

14. Lupton D, Jutel A. Soc Sci Med. 2015. 'It's like having a physician in your pocket!' A critical analysis of self-diagnosis smartphone apps URL: https://linkinghub.elsevier.com/retrieve/pii/S0277953615002245 [accessed 2019-03-13] [WebCite Cache ID 76qcHLZwk]

15. Ramo DE, Popova L, Grana R, Zhao S, Chavez K. Cannabis mobile apps: a content analysis. JMIR Mhealth Uhealth 2015 Aug 12;3(3):e81 [FREE Full text] [doi: 10.2196/mhealth.4405] [Medline: 26268634]

16. Bindhim NF, Naicker S, Freeman B, Mcgeechan K, Trevena L. Apps promoting illicit drugs-a need for tighter regulation? J Con Health Internet 2014 Feb 24;18(1):31-43 [ [FREE Full text] [doi: 10.1080/15398285.2014.869166]

17. Grundy QH, Wang Z, Bero LA. Challenges in assessing mobile health app quality: a systematic review of prevalent and innovative methods. Am J Prev Med 2016 Dec;51(6):1051-1059. [doi: 10.1016/j.amepre.2016.07.009] [Medline: 27659122]

18. Stoyanov SR, Hides L, Kavanagh DJ, Zelenko O, Tjondronegoro D, Mani M. Mobile app rating scale: a new tool for assessing the quality of health mobile apps. JMIR Mhealth Uhealth 2015;3(1):e27 [FREE Full text] [doi: 10.2196/mhealth.3422] [Medline: 25760773]

19. Jahns RG. Research2Guidance. 2015 Jan 1. 500m people will be using healthcare mobile applications in 2015 URL: https:/ /research2guidance.com/500m-people-will-be-using-healthcare-mobile-applications-in-2015-2/ [accessed 2019-03-13]

[WebCite Cache ID 76qczbyrL]

20. Saitz R, Cheng DM, Winter M, Kim TW, Meli SM, Allensworth-Davies D, et al. Chronic care management for dependence on alcohol and other drugs: the AHEAD randomized trial. J Am Med Assoc 2013 Sep 18;310(11):1156-1167 [FREE Full text] [doi: 10.1001/jama.2013.277609] [Medline: 24045740]

21. Weiss RD, Potter JS, Provost SE, Huang Z, Jacobs P, Hasson A, et al. A multi-site, two-phase, Prescription Opioid Addiction Treatment Study (POATS): rationale, design, and methodology. Contemp Clin Trials 2010 Mar;31(2):189-199 [FREE Full text] [doi: 10.1016/j.cct.2010.01.003] [Medline: 20116457]

22. Tofighi B, Grossman E, Bereket S. Text message content preferences to improve buprenorphine maintenance treatment in primary care. J Addict Dis 2016;35(2):92-100. [doi: 10.1080/10550887.2015.1127716] [Medline: 26670868]

23. Galanter M. Spirituality and recovery in 12-step programs: an empirical model. J Subst Abuse Treat 2007 Oct;33(3):265-272 [FREE Full text] [doi: 10.1016/j.jsat.2007.04.016] [Medline: 17889297]

24. Volkow ND, Collins FS. The role of science in addressing the opioid crisis. N Engl J Med 2017 Jul 27;377(4):391-394 [FREE Full text] [doi: 10.1056/NEJMsr1706626] [Medline: 28564549]

25. Tinschert P, Jakob R, Barata F, Kramer J, Kowatsch T. The potential of mobile apps for improving asthma self-management: a review of publicly available and well-adopted asthma apps. JMIR Mhealth Uhealth 2017 Aug 2;5(8):e113 [FREE Full text] [doi: 10.2196/mhealth.7177] [Medline: 28768606]

26. Athilingam P, Jenkins B. Mobile phone apps to support heart failure self-care management: integrative review. JMIR Cardio 2018 May 2;2(1):e10057 [FREE Full text] [doi: 10.2196/10057] 
27. Adam A, Hellig J, Perera M, Bolton D, Lawrentschuk N. 'Prostate Cancer Risk Calculator' mobile applications (apps): a systematic review and scoring using the validated user version of the Mobile Application Rating Scale (uMARS). World J Urol 2018 Apr;36(4):565-573. [doi: 10.1007/s00345-017-2150-1] [Medline: 29222595]

28. Mani M, Kavanagh DJ, Hides L, Stoyanov SR. Review and evaluation of mindfulness-based iPhone apps. JMIR Mhealth Uhealth 2015;3(3):e82 [FREE Full text] [doi: 10.2196/mhealth.4328] [Medline: 26290327]

29. BinDhim NF, Hawkey A, Trevena L. A systematic review of quality assessment methods for smartphone health apps. Telemed J E Health 2015 Feb;21(2):97-104. [doi: 10.1089/tmj.2014.0088] [Medline: 25469795]

30. Dallery J, Jarvis B, Marsch L, Xie H. Mechanisms of change associated with technology-based interventions for substance use. Drug Alcohol Depend 2015 May 1;150:14-23 [FREE Full text] [doi: 10.1016/j.drugalcdep.2015.02.036] [Medline: 25813268]

31. National Institute on Drug Abuse. Principles Of Drug Addiction Treatment: A Research-based Guide: Third Edition. Bethesda, Maryland: Createspace Independent Publishing Platform; 2018.

32. Volkow ND. Medications for opioid use disorder: bridging the gap in care. Lancet 2018 Dec 27;391(10118):285-287 [FREE Full text] [doi: 10.1016/S0140-6736(17)32893-3] [Medline: 29150199]

33. Lee JD, Vocci F, Fiellin DA. Unobserved. J Addict Med 2014;8(5):299-308. [doi: 10.1097/ADM.0000000000000059] [Medline: 25254667]

34. Lee JD, Grossman E, Huben L, Manseau M, McNeely J, Rotrosen J, et al. Extended-release naltrexone plus medical management alcohol treatment in primary care: findings at 15 months. J Subst Abuse Treat 2012 Dec;43(4):458-462 [FREE Full text] [doi: 10.1016/j.jsat.2012.08.012] [Medline: 22985676]

35. Anderson P, Scott E. The effect of general practitioners' advice to heavy drinking men. Br J Addict 1992 Jun;87(6):891-900 [FREE Full text] [doi: 10.1111/j.1360-0443.1992.tb01984.x] [Medline: 1525531]

36. Spivak K, Sanchez-Craig M, Davila R. Assisting problem drinkers to change on their own: effect of specific and non-specific advice. Addiction 1994 Sep;89(9):1135-1142 [FREE Full text] [doi: 10.1111/j.1360-0443.1994.tb02790.x] [Medline: 7987190]

37. Kelly PJ, Deane FP, Baker AL. Group cohesion and between session homework activities predict self-reported cognitive-behavioral skill use amongst participants of SMART Recovery groups. J Subst Abuse Treat 2015 Apr;51:53-58 [FREE Full text] [doi: 10.1016/j.jsat.2014.10.008] [Medline: 25535099]

38. Methadone.US. URL: http://www.methadone.us/ [accessed 2019-03-15] [WebCite Cache ID 76tD6vzuy]

39. Crane D, Garnett C, Brown J, West R, Michie S. Behavior change techniques in popular alcohol reduction apps: content analysis. J Med Internet Res 2015;17(5):e118 [FREE Full text] [doi: 10.2196/jmir.4060] [Medline: 25977135]

40. Masterson Creber RM, Maurer M, Reading M, Hiraldo G, Hickey K, Iribarren S. Review and analysis of existing mobile phone apps to support heart failure symptom monitoring and self-care management using the Mobile Application Rating Scale (MARS). JMIR Mhealth Uhealth 2016 Jun 14;4(2):e74 [FREE Full text] [doi: 10.2196/mhealth.5882] [Medline: 27302310]

41. Gonzalez VM, Dulin PL. Comparison of a smartphone app for alcohol use disorders with an internet-based intervention plus bibliotherapy: a pilot study. J Consult Clin Psychol 2015 Apr;83(2):335-345. [doi: 10.1037/a0038620] [Medline: 25622202]

42. Dulin PL, Gonzalez VM. Smartphone-based, momentary intervention for alcohol cravings amongst individuals with an alcohol use disorder. Psychol Addict Behav 2017 Dec;31(5):601-607 [FREE Full text] [doi: 10.1037/adb0000292] [Medline: 28703611]

43. Shuren J, Patel B, Gottlieb S. FDA regulation of mobile medical apps. J Am Med Assoc 2018 Jul 24;320(4):337-338. [doi: 10.1001/jama.2018.8832] [Medline: 29971339]

44. BinDhim NF, Freeman B, Trevena L. Pro-smoking apps for smartphones: the latest vehicle for the tobacco industry? Tob Control 2014 Jan;23(1):e4. [doi: 10.1136/tobaccocontrol-2012-050598] [Medline: 23091161]

45. Steinberg J. Inc Magazine. 2018 Feb 1. Google removed 700,000 problematic apps From its android play store in 2017 URL: https://www.inc.com/joseph-steinberg/google-removed-700000-problematic-apps-from-its-android-play-store-in-2017. html [accessed 2019-03-13] [WebCite Cache ID 76qdn7jVN]

46. Kellogg S, Kreek MJ. On blending practice and research: the search for commonalities in substance abuse treatment. Subst Abus 2006 Jun;27(1-2):9-24 [FREE Full text] [doi: 10.1300/J465v27n01_03] [Medline: 17062541]

47. Tofighi B, Grossman E, Sherman S, Nunes EV, Lee JD. Mobile Phone Messaging During Unobserved. J Addict Med 2016;10(5):309-313. [doi: 10.1097/ADM.0000000000000198] [Medline: 26933874]

\section{Abbreviations}

ASSIST: Alcohol, Smoking and Substance Involvement Screening Test

AUDIT: Alcohol Use Disorders Identification Test

CBT: Cognitive Behavioral Therapy

FDA: Food and Drug Administration

HCV: hepatitis C virus 
ICC: intraclass correlation

MARS: Mobile App Rating Scale

mHealth: mobile health

OBOT: Office based opioid treatment

OUD: Opioid use disorder

SAMHSA: Substance Abuse and Mental Health Services Administration

SMART: Self-Management and Recovery Training

SMS: short message service

SUD: substance use disorder

Edited by G Eysenbach; submitted 03.08.18; peer-reviewed by P Dulin, J Glass, D Gustafson, A Carr; comments to author 07.10.18; revised version received 24.10.18; accepted 12.12.18; published 22.04.19

Please cite as:

Tofighi B, Chemi C, Ruiz-Valcarcel J, Hein P, Hu L

Smartphone Apps Targeting Alcohol and Illicit Substance Use: Systematic Search in in Commercial App Stores and Critical Content Analysis

JMIR Mhealth Uhealth 2019;7(4):e11831

URL: https://mhealth.jmir.org/2019/4/e11831/

doi: $\underline{10.2196 / 11831}$

PMID: 31008713

(CBabak Tofighi, Chemi Chemi, Jose Ruiz-Valcarcel, Paul Hein, Lu Hu. Originally published in JMIR Mhealth and Uhealth (http://mhealth.jmir.org), 22.04.2019. This is an open-access article distributed under the terms of the Creative Commons Attribution License (https://creativecommons.org/licenses/by/4.0/), which permits unrestricted use, distribution, and reproduction in any medium, provided the original work, first published in JMIR mhealth and uhealth, is properly cited. The complete bibliographic information, a link to the original publication on http://mhealth.jmir.org/, as well as this copyright and license information must be included. 